\title{
Application of IRDF2002 dosimetry data to shielding benchmarks using the Monte Carlo code MCBEND
}

\author{
G.A. Wright ${ }^{1}$ and C.J. Dean ${ }^{1, a}$ \\ Serco Assurance, Winfrith Technology Centre, Dorchester DT2 8DH, UK
}

\begin{abstract}
The IRDF2002 dosimetry cross section data are processed into very fine energy groups and added to the detector response library for the Monte Carlo code MCBEND. The IRDF2002 covariances are processed into broad groups to form a MCBEND detector covariance library. A number of benchmark experiments are analysed using MCBEND with both IRDF2002 and IRDF90 data and the results compared. Uncertainties due to material and detector cross sections are calculated automatically. The results demonstrate that the IRDF2002 ${ }^{115} \operatorname{In}\left(\mathrm{n}, \mathrm{n}^{\prime}\right)^{115 \mathrm{~m}} \mathrm{In}$ dosimetry cross sections give improved agreement between calculation and measurement in an iron benchmark and that use of IRDF2002 data allows straightforward, explicit and accurate calculation of single resonance detector reaction-rates, e.g., ${ }^{197} \mathrm{Au}(\mathrm{n}, \gamma){ }^{198} \mathrm{Au}$.
\end{abstract}

\section{Introduction}

Nuclear data form an integral part of dosimetry analyses: neutron transport is determined by material nuclear data (cross sections and secondary angle/energy distributions) and indicated by detector reaction-rates which are also dependent on detector cross sections. Thus improvements in detector cross sections may have a significant effect on such analyses. The uncertainties on detector reaction-rates due to uncertainties in the material data and detector cross sections are also fundamental to dosimetry.

Generally one of the purposes of dosimetry analyses is to benchmark computer codes and nuclear data by comparing calculated and measured detector reaction-rates. These can be on reactor plant or in material benchmark experiments such as those contained in the SINBAD database [1]. The results may be used to assign correction factors and also uncertainty when predicting quantities such as displacements per atom (dpa) using the same computer code and data. Improvements in detector cross sections will help to improve the agreement between calculated and measured reaction-rates thus reducing correction factors and assigned uncertainties.

Uncertainties on material cross sections will affect both detector reaction-rates and quantities such as dpa but those on detector cross sections will only affect the detector results. This should be taken into account when assigning correction factors or uncertainties. Traditionally computation of uncertainty on detector reaction-rates has been a somewhat laborious process and thus the domain of the expert rather than the regular analyst. Hence automatic calculation of the contributions to this uncertainty is an important step forward in making uncertainty analysis more accessible.

One particular difficulty for the dosimetry analyst is the accurate calculation of reaction-rates for detectors that contain large resonances, such as ${ }^{55} \mathrm{Mn}(\mathrm{n}, \gamma)^{56} \mathrm{Mn}$ and ${ }^{197} \mathrm{Au}$ $(\mathrm{n}, \gamma)^{198} \mathrm{Au}$. The resonance causes the flux to be suppressed significantly and this suppression needs to be taken into

\footnotetext{
a Presenting author,

e-mail: christopher.dean@sercoassurance.com
}

account in the calculation. This can be done either by applying a dilute cross section and modelling the foil in its environment or by applying a suppressed cross section and not including the foil. Adequate resolution of the resonance is important.

\section{The IRDF2002 dosimetry library}

The IRDF2002 dosimetry library has recently been released by the IAEA [2]. The library was formed following a thorough review of existing and new evaluations of dosimetry data. The sources used for the library were: IRDF90, JENDL/D-99 and updates, RRDF-98 and updates, ENDF/B-VI and JEFF3.0. There are around 80 reactions on the library, including some damage reactions.

For most reactions the library contains point energy cross sections as well as cross sections in the SAND-IIa 640 energy group scheme used for the IRDF90 library. Use of the point energy data allows the possibility of more accurate representation of detector cross sections for use in applications codes. This is important for threshold reactions in deep penetration problems and for resonance reactions. The IRDF2002 library also contains covariance data for the majority of reactions.

\section{Production of detector cross section and covariance libraries}

The IRDF2002 data have been processed using NJOY [3] to produce a detector cross section library and a detector covariance library for use with the Monte Carlo code MCBEND [4]. The detector cross sections are added to an existing library whilst the covariance library is new.

Since the point detector cross sections from IRDF2002 assume linear variation between points and are at room temperature they were processed into 13,230 energy groups as shown in table 1 using the NJOY modules MODER and GROUPR. This energy group scheme is fine enough to account for resonance self shielding of single resonance 
Table 1. Energy group scheme for MCBEND detector response library.

\begin{tabular}{ccc}
\hline Energy range & $\begin{array}{c}\text { Number } \\
\text { of groups }\end{array}$ & Comments \\
\hline $0 \mathrm{eV}-73 \mathrm{eV}$ & 7217 & equal energy or lethargy \\
$73 \mathrm{eV}-10 \mathrm{keV}$ & 5040 & $1 / 1024$ lethargy \\
$10 \mathrm{keV}-20 \mathrm{MeV}$ & 973 & $1 / 128$ lethargy \\
\hline
\end{tabular}

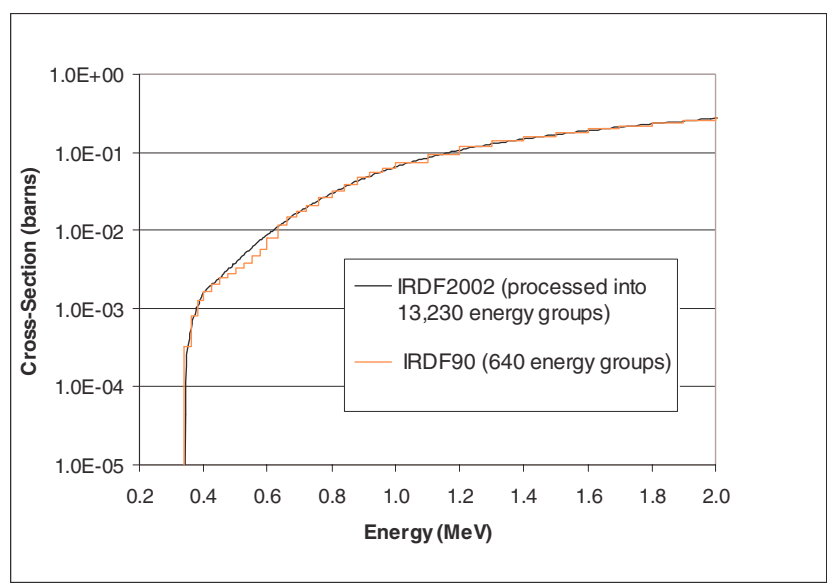

Fig. 1. Comparison of IRDF2002 and IRDF90 ${ }^{115} \operatorname{In}\left(\mathrm{n}, \mathrm{n}^{\prime}\right)^{115 \mathrm{~m}} \mathrm{In}$ dosimetry cross sections.

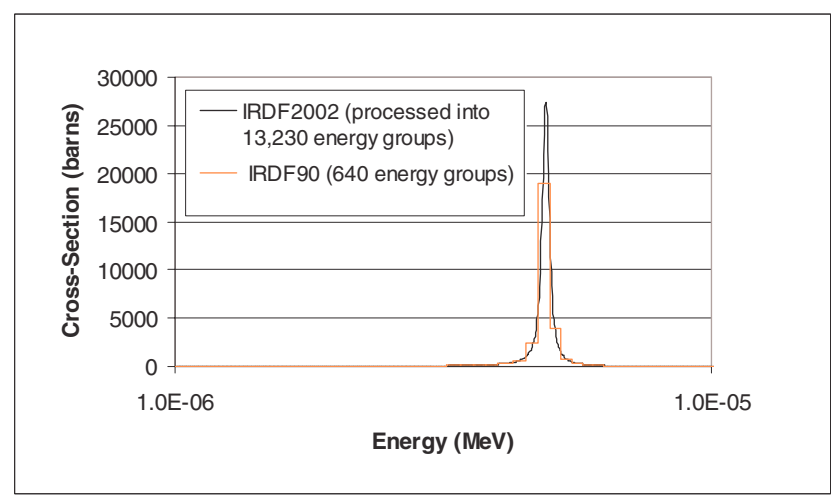

Fig. 2. Comparison of IRDF2002 and IRDF90 ${ }^{197} \mathrm{Au}(\mathrm{n}, \gamma){ }^{198} \mathrm{Au}$ dosimetry cross sections.

reactions and also gives a good representation of threshold reactions.

Comparisons between IRDF2002 and IRDF90 cross sections for the ${ }^{115} \mathrm{In}\left(\mathrm{n}, \mathrm{n}^{\prime}\right){ }^{115 \mathrm{~m}} \mathrm{In}$ and ${ }^{197} \mathrm{Au}(\mathrm{n}, \gamma){ }^{198} \mathrm{Au}$ reactions are shown in figure 1 and figure 2 . For the ${ }^{115} \operatorname{In}\left(n, n^{\prime}\right)^{115 m} \operatorname{In}$ reaction the processed IRDF2002 data provide improved resolution in the threshold region and are also different from the IRDF90 data in part of that region. For the ${ }^{197} \mathrm{Au}(\mathrm{n}, \gamma){ }^{198} \mathrm{Au}$ reaction it can be seen that the processed IRDF2002 data provide adequate resolution of the resonance (17 groups cover the resonance width at half height) whereas the IRDF90 data do not (one group covers the resonance width at half height). Thus the dilute IRDF90 data could not be used for accurate prediction of this reaction-rate and recourse would have to be made to using fine group material data or to using a suppressed cross section.
The covariance data from IRDF2002 were processed into 25 broad energy groups using the NJOY modules MODER, ERRORR and COVR. Any short range variance data $(\mathrm{LB}=8)$ were suppressed by means of the negative MATNO flag which is now available in ERRORR.

\section{Uncertainty analysis in the Monte Carlo code MCBEND}

MCBEND is a general geometry, point energy, Monte Carlo code used for radiation transport calculations for neutrons, gamma-rays and electrons that is available from the ANSWERS Software Service. MCBEND has been extensively applied to dosimetry analysis for UK gas cooled reactors and to PWR and BWR reactors [5]. A large amount of benchmarking against single and multimaterial experiments has also been undertaken [6]. Material cross sections are held in 13,193 energy groups (as table 1 but from $0-15 \mathrm{MeV}$ ) and secondary angle and energy distributions are also accurately represented. Detector cross sections are available in a detector response library and libraries of material and detector covariances are available.

MCBEND automatically calculates the uncertainties on the detector reaction-rate due to uncertainties in the material data and due to uncertainties in detector cross sections. Sensitivities to material and detector cross sections are calculated during the calculation and these are then folded with covariance matrices read from the MCBEND material covariance library and the MCBEND detector covariance library. The sensitivities to material cross sections are calculated using a differential method and those to detector cross sections are simply the fractional contributions to the reaction-rate from each energy group. This facility is very useful and allows the analyst to calculate uncertainties with a minimum of additional work, thus making uncertainty analysis more accessible.

\section{Analysis of benchmarks}

MCBEND has been applied to a number of shielding benchmarks using both IRDF90 and IRDF2002 dosimetry data. Many of these were performed in the ASPIS facility at Winfrith and are included in the SINBAD database. One benchmark will be discussed in detail here: a single material iron benchmark.

\subsection{Iron benchmark}

The ASPIS shielding facility was installed on the NESTOR reactor at Winfrith. NESTOR was a light water cooled, graphite and light water moderated reactor which operated at powers of up to $30 \mathrm{~kW}$ and was used as a source of neutrons for a wide range of applications. Shield components, which were in the main slabs or tanks, were mounted vertically in a mobile tank which had an internal cross sectional area of $1.8 \mathrm{~m} \times 1.9 \mathrm{~m}$ and a length of $3.7 \mathrm{~m}$. A fission plate was located 
within the experimental shield array. The loaded tank was moved into the cave where thermal neutrons leaking from the outer graphite reflector of NESTOR were used to drive the fission plate to provide a well defined neutron source for penetration measurements. The absolute source strength was determined to a precision of $4 \%$ by fission product counting and the spatial distribution was determined via detailed low energy flux mapping with activation detectors.

The iron benchmark experimental array is shown schematically in side elevation in figure 3. The array comprised three regions; the source region containing moderator and the fission plate, the shield made from 13 mild steel plates, each of approximately $5.1 \mathrm{~cm}$ thickness, and a deep backing shield manufactured from mild and stainless steel. To allow detector access within the shield $6 \mathrm{~mm}$ spacers were placed between each plate. Measurements of the threshold reaction rates $\left.{ }^{32} \mathrm{~S}(\mathrm{n}, \mathrm{p})\right)^{32} \mathrm{P},{ }^{115} \mathrm{In}\left(\mathrm{n}, \mathrm{n}^{\prime}\right){ }^{115 \mathrm{~m}} \mathrm{In},{ }^{103} \mathrm{Rh}\left(\mathrm{n}, \mathrm{n}^{\prime}\right){ }^{103 \mathrm{~m}} \mathrm{Rh}$ and the epithermal ${ }^{197} \mathrm{Au}(\mathrm{n}, \gamma){ }^{198} \mathrm{Au} / \mathrm{Cd}$ reaction-rate were made in activation foils along the nuclear centre line.

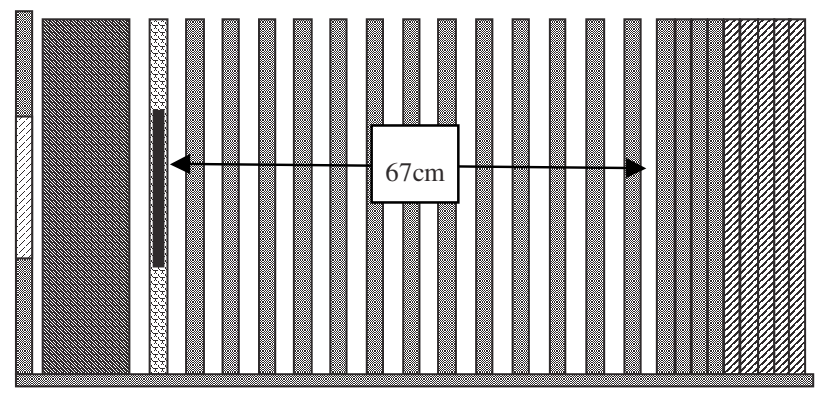

All components are $182.9 \mathrm{~cm}$ wide by $191.0 \mathrm{~cm}$ high Not To Scale

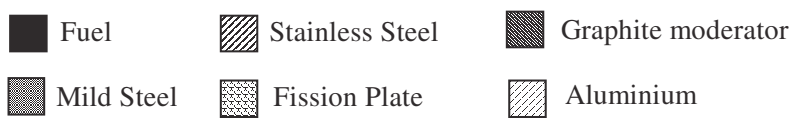

Fig. 3. Schematic diagram of iron benchmark.

\subsection{Threshold detector results}

The results show that there is no significant difference between results for the ${ }^{32} \mathrm{~S}(\mathrm{n}, \mathrm{p})^{32} \mathrm{P}$ reaction-rate obtained using IRDF2002 and IRDF90 data. For the ${ }^{103} \mathrm{Rh}\left(\mathrm{n}, \mathrm{n}^{\prime}\right)^{103 \mathrm{~m}} \mathrm{Rh}$ reaction-rate there is a slight reduction in reaction-rate with a difference of around $6 \%$ after $67 \mathrm{~cm}$ of iron. However, this is within the uncertainty due to the detector at this location (10\%) so is not significant. Hence for these two reactions the improved resolution of the threshold region has not produced significant differences in the predicted detector reaction-rates. Figure 4 shows the comparison of calculated and measured reaction-rates for the ${ }^{115} \operatorname{In}\left(n, n^{\prime}\right)^{115 m} I n$ reaction. It can be seen that moving to IRDF2002 data produces an increase in reaction-rate that improves agreement between calculation and measurement. The increase is greater than the uncertainty due to the detector so is significant. This difference is thought to be mainly due to the difference in the underlying cross section, as shown in figure 1, with the improved resolution of the threshold region being only a secondary effect.

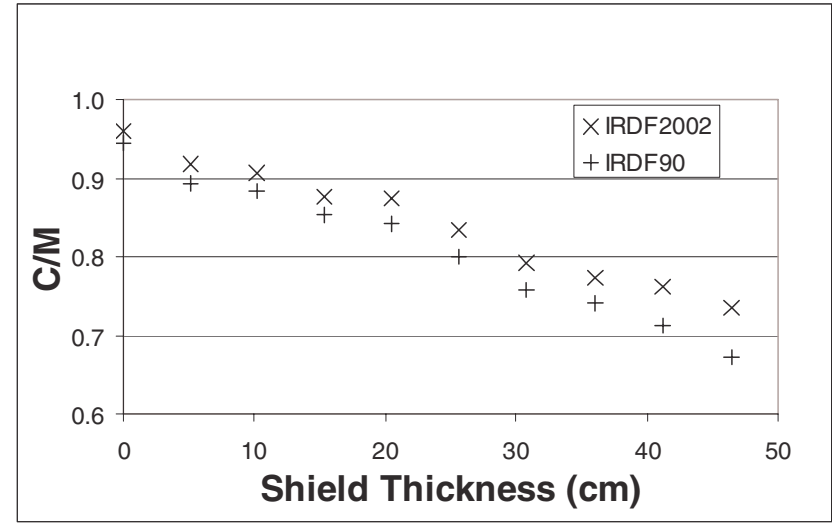

Fig. 4. Comparison of calculated and measured ${ }^{115} \operatorname{In}\left(n, n^{\prime}\right)^{115 m} \operatorname{In}$ reaction-rates in the iron benchmark.

Table 2. Uncertainties in the iron benchmark.

\begin{tabular}{|c|c|c|c|c|c|c|c|}
\hline 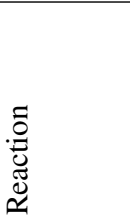 & 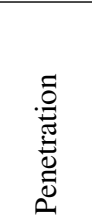 & 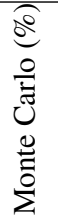 & 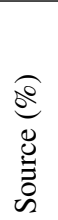 & 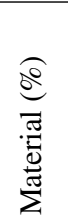 & 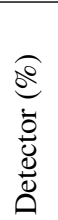 & $\frac{\sqrt{2}}{\frac{\pi}{0}}$ & $\sum_{U}$ \\
\hline \multirow[t]{3}{*}{ Sulphur } & $0 \mathrm{~cm}$ & 0.4 & 4.0 & 0.6 & 3.2 & 5.2 & 0.83 \\
\hline & $26 \mathrm{~cm}$ & 0.6 & 4.0 & 10.6 & 2.6 & 11.6 & 0.93 \\
\hline & $46 \mathrm{~cm}$ & 0.8 & 4.0 & 19.4 & 2.6 & 20.0 & 0.97 \\
\hline \multirow[t]{3}{*}{ Indium } & $0 \mathrm{~cm}$ & 0.3 & 4.0 & 0.7 & 1.7 & 4.4 & 0.96 \\
\hline & $26 \mathrm{~cm}$ & 0.4 & 4.0 & 5.6 & 2.0 & 7.2 & 0.83 \\
\hline & $46 \mathrm{~cm}$ & 0.5 & 4.0 & 7.4 & 2.4 & 8.8 & 0.74 \\
\hline \multirow[t]{3}{*}{ Rhodium } & $0 \mathrm{~cm}$ & 0.2 & 4.0 & 0.6 & 4.1 & 5.8 & 0.97 \\
\hline & $26 \mathrm{~cm}$ & 0.2 & 4.0 & 2.7 & 5.4 & 7.2 & 0.93 \\
\hline & $46 \mathrm{~cm}$ & 0.3 & 4.0 & 3.8 & 7.3 & 9.2 & 0.85 \\
\hline
\end{tabular}

Uncertainties (given as percentages) are quoted at the 1 s.d. level.

Table 2 shows the results of uncertainty analysis for shielding penetrations of $0 \mathrm{~cm}, 26 \mathrm{~cm}$ and $46 \mathrm{~cm}$ for the threshold detectors. The ratios of calculated to measured reaction-rates are also given. For the ${ }^{32} \mathrm{~S}(\mathrm{n}, \mathrm{p})^{32} \mathrm{P}$ and ${ }^{115} \mathrm{In}\left(\mathrm{n}, \mathrm{n}^{\prime}\right)^{115 \mathrm{~m}} \mathrm{In}$ reactions the uncertainty at deep penetration is dominated by that due to the material cross sections whilst for the ${ }^{103} \mathrm{Rh}\left(\mathrm{n}, \mathrm{n}^{\prime}\right)^{103 \mathrm{~m}} \mathrm{Rh}$ reaction the detector cross section uncertainty dominates, due to the increased importance of the threshold region at deep penetration in this system in which the spectrum softens with penetration.

\subsection{Epithermal detector results}

The calculation of the ${ }^{197} \mathrm{Au}(\mathrm{n}, \gamma){ }^{198} \mathrm{Au} / \mathrm{Cd}$ reaction-rate has been carried out with the thickness of the gold foil ( 2 thousandths of an inch) included in the model and with dilute cross sections applied. Previously this reaction-rate was calculated without the foil modelled but using a suppressed cross section. This suppressed cross section was derived very carefully from analysis of a stainless steel benchmark experiment and in the resonance region was represented in the 640 group SANDIIa scheme. A finer representation was not possible due to the difficulty of scoring fluxes sufficiently precisely in such a scheme. In the stainless steel benchmark this suppressed cross 
section was found to agree with the reaction-rate obtained using a 13,193 group cross section extracted from the JEF2.2 library (from which it was derived) to within $8 \%$, with a mean difference of around $3 \%$, which was considered to be acceptable.

The comparison of calculated and measured reaction-rates obtained using the dilute IRDF2002 cross section with foil modelled and the suppressed cross section with no foil modelled for the iron benchmark is shown in figure 5. The difference is within $18 \%$ with the mean difference around $7 \%$, demonstrating that the suppressed cross section is, as one would expect, not universally applicable. The IRDF2002 data give improved agreement with experiment. Hence the introduction of IRDF2002 point energy cross sections, processed into a very fine energy group scheme, allows the ${ }^{197} \mathrm{Au}(\mathrm{n}, \gamma){ }^{198} \mathrm{Au}$ reaction-rate to be readily calculated explicitly and accurately using the available dosimetry library.

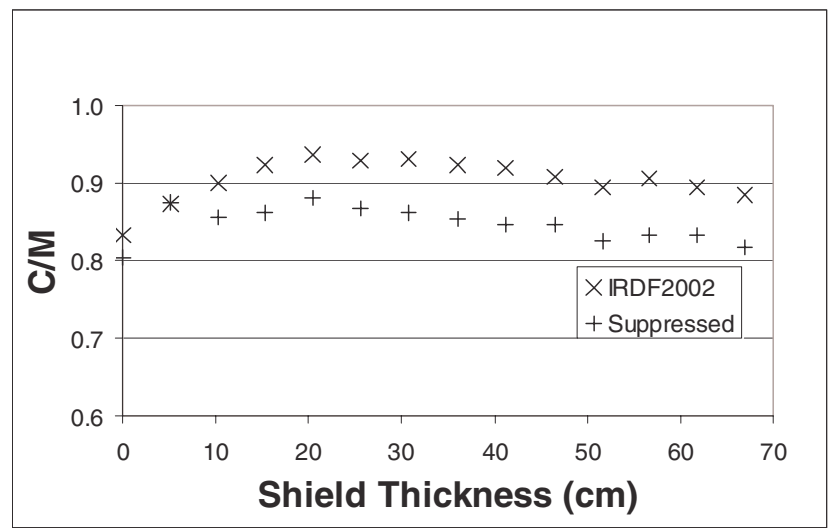

Fig. 5. Comparison of calculated and measured ${ }^{197} \mathrm{Au}(\mathrm{n}, \gamma){ }^{198} \mathrm{Au} / \mathrm{Cd}$ reaction-rates in the iron benchmark.

\subsection{Other benchmarks}

Various other SINBAD benchmarks are also being analysed : graphite, iron/water, graphite/steel and the HB Robinson PWR. A synopsis of the results obtained is given here.

For the graphite benchmark there are no significant differences between results for ${ }^{32} \mathrm{~S}(\mathrm{n}, \mathrm{p})^{32} \mathrm{P},{ }^{115} \mathrm{In}\left(\mathrm{n}, \mathrm{n}^{\prime}\right)^{115 \mathrm{~m}} \mathrm{In}$ and ${ }^{103} \mathrm{Rh}\left(\mathrm{n}, \mathrm{n}^{\prime}\right){ }^{103 \mathrm{~m}} \mathrm{Rh}$ reaction-rates obtained with IRDF2002 and IRDF90 dosimetry data. This is because in this system the spectrum hardens with penetration so the threshold region becomes less important. For the iron/water and graphite/steel benchmarks the results are similar to those for the iron benchmark: there are no significant differences between
${ }^{32} \mathrm{~S}(\mathrm{n}, \mathrm{p}){ }^{32} \mathrm{P}$ and ${ }^{103} \mathrm{Rh}\left(\mathrm{n}, \mathrm{n}^{\prime}\right){ }^{103 \mathrm{~m}} \mathrm{Rh}$ reaction-rates obtained using IRDF2002 and IRDF90 data but there are some differences for ${ }^{115} \operatorname{In}\left(\mathrm{n}, \mathrm{n}^{\prime}\right)^{115 \mathrm{~m}} \mathrm{In}$ reaction-rates.

For the HB Robinson PWR benchmark a variety of reactor dosimetry detector reactions are being used. There are significant differences of a few percent between results in the reactor cavity for ${ }^{58} \mathrm{Ni}(\mathrm{n}, \mathrm{p}){ }^{58} \mathrm{Co},{ }^{63} \mathrm{Cu}(\mathrm{n}, \alpha){ }^{60} \mathrm{Co},{ }^{59} \mathrm{Co}(\mathrm{n}, \gamma){ }^{60} \mathrm{Co}$ and ${ }^{237} \mathrm{~Np}(\mathrm{n}, \mathrm{f})$ whilst the ${ }^{46} \mathrm{Ti}(\mathrm{n}, \mathrm{p}){ }^{46} \mathrm{Sc}$ results show a significant difference of $15 \%$. Other reactions such as ${ }^{54} \mathrm{Fe}(\mathrm{n}, \mathrm{p}){ }^{54} \mathrm{Mn}$, ${ }^{238} \mathrm{U}(\mathrm{n}, \mathrm{f})$ and ${ }^{235} \mathrm{U}(\mathrm{n}, \mathrm{f})$ do not exhibit significant differences. Thus introduction of IRDF2002 dosimetry data may cause some differences in reactor dosimetry results, although in general these are not expected to be large.

\section{Conclusions}

The recently released IRDF2002 dosimetry library has been processed to produce detector cross sections for use in the Monte Carlo code MCBEND in 13,230 energy groups together with a detector covariance library in 25 energy groups. These have been used to analyse a number of benchmarks and the results compared against those obtained using IRDF90 data. Uncertainties, automatically calculated by MCBEND, are taken into account. The IRDF2002 ${ }^{115} \operatorname{In}\left(\mathrm{n}, \mathrm{n}^{\prime}\right)^{115 \mathrm{~m}} \mathrm{In}$ dosimetry cross sections give improved agreement between calculation and measurement in an iron benchmark. Use of IRDF2002 data allows straightforward, explicit and accurate calculation of single resonance detector reaction-rates, such as ${ }^{197} \mathrm{Au}(\mathrm{n}, \gamma){ }^{198} \mathrm{Au}$, eliminating the need to consider resonance shielding separately.

\section{References}

1. I. Kodeli et al., in Proceedings of the $14^{\text {th }}$ Biennial Topical Meeting of the Radiation Protection and Shielding Division, Carlsbad, New Mexico, 2006 (ANS, 2006), pp. 87-92.

2. International Reactor Dosimetry File 2002 (IRDF-2002), IAEA Technical Reports Series No. 452, 2006.

3. R.E. MacFarlane, D.W. Muir, Los Alamos Report No. LA-12740M, 1994.

4. P. Cowan et al., Radiat. Prot. Dosim. 115, 403 (2005).

5. G.A. Wright et al., in Reactor Dosimetry, edited by J.G. Williams et al. (ASTM, Pennsylvania, 2000), pp. 540-548.

6. G.A. Wright et al., in Proceedings of the $8^{\text {th }}$ International Conference on Radiation Shielding, Arlington, Texas, 1994 (ANS, Illinois, 1994), pp. 816-823. 